\title{
The effect of host genetics on in vitro performance of bovine monocyte-derived macrophages
}

\author{
Mehdi Emam, ${ }^{1,2 *}$ Saeid Tabatabaei, ${ }^{1}$ Mehdi Sargolzaei, ${ }^{1,3}$ Shayan Sharif, ${ }^{1}$ Flavio Schenkel, ${ }^{2}$ \\ and Bonnie Mallard ${ }^{1,2}$ \\ ${ }^{1}$ Department of Pathobiology, Ontario Veterinary College, University of Guelph, Guelph, Ontario, N1G 2W1, Canada \\ ${ }^{2}$ Center for Genetic Improvement of Livestock, Department of Animal Bioscience, University of Guelph, Guelph, Ontario, N1G 2W1, Canada \\ ${ }^{3}$ Select Sires Inc., Plain City, $\mathrm{OH} 43064$
}

\section{ABSTRACT}

The dynamic interaction between the host and pathogens, along with environmental factors, influences the regulation of mammalian immune responses. Therefore, comprehensive in vivo immune-phenotyping during an active response to a pathogen can be complex and prone to confounding effects. Evaluating critical fundamental aspects of the immune system at a cellular level is an alternative approach to reduce this complexity. Therefore, the objective of the current study was to examine an in vitro model for functional phenotyping of bovine monocyte-derived macrophages (MDM), cells which play a crucial role at all phases of inflammation, as well influence downstream immune responses. As indicators of MDM function, phagocytosis and nitric oxide $\left(\mathrm{NO}^{-}\right)$production were tested in MDM of 16 cows in response to 2 common bacterial pathogens of dairy cows, Escherichia coli and Staphylococcus aureus. Notable functional variations were observed among the individuals (coefficient of variation: $33 \%$ for phagocytosis and $70 \%$ in the production of $\mathrm{NO}^{-}$). The rank correlation analysis revealed a significant, positive, and strong correlation (rho $=0.92$ ) between $\mathrm{NO}^{-}$production in response to $E$. coli and $S$. aureus, and a positive but moderate correlation $(\mathrm{rho}=0.58)$ between phagocytosis of E. coli and S. aureus. To gain further insight into this trait, another 58 cows were evaluated solely for $\mathrm{NO}^{-}$response against $E$. coli. The pedigree of the tested animals was added to the statistical model and the heritability was estimated to be 0.776 . Overall, the finding of this study showed a strong effect of host genetics on the in vitro activities of MDM and the possibility of ranking Holstein cows based on the in vitro functional variation of MDM.

Key words: monocyte-derived macrophage, phagocytosis, nitric oxide production, rank correlation

Received November 7, 2018.

Accepted May 17, 2019.

${ }^{*}$ Corresponding author: semam@uoguelph.ca

\section{INTRODUCTION}

Genetic regulation of immune responses in mammals is exceptionally complex with about $18 \%$ of the human genome (7,696 genes) annotated with the "immune response" term. In the bovine genome, this proportion increases to $23 \%$ with the total number of genes as 5,586 (ENSEMBL, release 93; Zerbino et al., 2018). Analyzing host resistance or resilience in natural or experimental challenge models has been widely used to understand the genetic regulation of disease resistance (Min-Oo et al., 2007; Longley et al., 2011; Bishop, 2012; McManus et al., 2014; Abel et al., 2018). However, often the dynamic interaction between the host and pathogen, as well as the environmental effects which have a substantial effect on the outcome of infection, have resulted in various findings, which shows the need for a well-defined phenotype for disease resistance (Minozzi et al., 2012; Greives et al., 2017). An alternative approach is to reduce the complexity of this system by removing the effects of the pathogen by measuring host immunocompetence without pathogen exposure (Thompson-Crispi et al., 2012b; Greives et al., 2017). This approach has revealed a substantial contribution of host genetics in the variation of bovine immune responses. The heritabilities of immunocompetence traits in cattle have been estimated to be approximately 3 - to 4 -fold larger than the heritability of disease occurrence and immune responses against specific pathogens (Clapperton et al., 2009; Ring et al., 2018). Evaluating key cellular components of the immune system in vitro is another way to reduce the complexity of immunocompetence testing. Recently, immunophenotyping based on the performance of the cells of immune system in in vitro models has been successfully carried out in humans. These cellular immunogenetics studies have identified phenotypic variation in cellular responses which then were linked to genetic variations and cellular mechanisms that control the resistance to bacterial infection in human populations (Ko et al., 2013; Alvarez et al., 2017; Wang et al., 2018). In cattle, 
immunophenotyping based on in vivo responses of the adaptive immune system is currently available. Studies have shown that the phenotypes of adaptive immune responses are linked to the naturally occurring genetic variation and associated with resistance to infectious diseases (Thompson-Crispi et al., 2012a, 2014; Mallard et al., 2015). However, the innate arm of the immune system has remained less investigated and there is still a need to identify a robust model to further the understanding of genetic regulation of the innate responses in cattle. Identifying phenotypic variation in functional traits is the primary requirement to identify and understand the genetic mechanisms that shape the trait. As an initial step toward this goal, it was hypothesized that there is considerable variation in the function of bovine monocyte-derived macrophages (MDM) following in vitro exposure to bacterial pathogens and that it would be possible to identify individuals which respond stronger than others. Macrophages are a key component of the innate immune system that play a crucial role in the early phase of inflammation (Dunster, 2016; Hamidzadeh et al., 2017). Therefore, the objective of this study was to examine an in vitro model of phenotyping bovine MDM, and to evaluate phenotypic and genetic variance. As indicators of MDM function, phagocytosis and nitric oxide $\left(\mathrm{NO}^{-}\right)$production were evaluated following exposure of MDM to 2 common bacterial pathogens of dairy cows, Escherichia coli and Staphylococcus aureus.

\section{MATERIALS AND METHODS}

\section{Animals}

Cows for this study were selected from the research herd at the University of Guelph. This research herd is approximately 4 times larger than the average commercial dairy herds in Canada. All the cows are registered with Holstein Canada, and health records and treatments are recorded in detail. Hence, samples from this herd provided genetic diversity as well as detailed environmental records for the statistical analysis. The pedigree of the herd was obtained from the Canadian Dairy Network and the relationship between the cows was tested and full-sib animals were removed from the study to maximize host genetic variation of the samples. Sixteen milking cows that were offspring of 12 sires, 15 dams, 9 paternal grandsires, and 14 maternal grandsires were selected. These animals were not diagnosed or treated for any diseases in the lactation period in which samples were collected. Samples were collected in groups of 4 cows per sampling day. For the second part of the study, an additional 58 samples (offspring of 33 sires, 57 dams, 23 paternal grandsires, and 37 maternal grandsires) were collected from the same barn with similar criteria to calculate genetic parameters, specifically the heritability of $\mathrm{MDM} \mathrm{NO}^{-}$response. All the procedures and handling of the animals were approved by the animal care committee of the University of Guelph.

\section{In Vitro Transformation of Monocyte to Macrophages}

Blood samples were collected from the tail vein in tubes containing EDTA. Blood mononuclear cells (BMC) were purified based on the gradient centrifuge separation method. Concisely, Histopaque-1077 (Sigma-Aldrich, St. Louis, MO) was loaded into the Sepmate tubes (Stemcell Technologies, Vancouver, BC, Canada) and whole-blood samples were overlaid on the top of Histopaque-1077. After centrifugation for $10 \mathrm{~min}$ at $1,200 \times g$ at room temperature, the layer of cells above the Histopaque-1077 was collected and washed 3 times to obtain the purified BMC. Purified BMC were cultured at a concentration of $1 \times 10^{6}$ cells per square centimeter of the culture flask for $2 \mathrm{~h}$ in Monocyte Attachment Medium (PromoCell, Heidelberg, Germany) at $37^{\circ} \mathrm{C}$. Nonadherent cells were removed by washing and the medium was replaced with AIM V medium (Thermo Fisher Scientific Inc., Mississauga, ON, Canada) containing $5 \mathrm{ng} / \mathrm{mL}$ of recombinant bovine granulocyte-macrophage colony-stimulating factor (GM-CSF, Kingfisher Biotech, St. Paul, MN; chosen based on maximizing the number of harvested cells at the lowest concentration of GM-CSF in a titration experiment using $2.5,5$, and $10 \mathrm{ng} / \mathrm{mL}$ ) in the presence of $5 \% \mathrm{CO}_{2}$. After $6 \mathrm{~d}$ of incubation, adherent cells were detached from the flask using TrypLE Select Enzyme (Thermo Fisher Scientific Inc.). Phenotypic characteristics of macrophages (CD14 ${ }^{+}, \mathrm{CD} 205^{-}$, and strong auto-fluorescence) were analyzed using flow cytometry to determine the proportion of macrophages among the harvested cells (Njoroge et al., 2001; Fuentes-Duculan et al., 2010; Mitchell et al., 2010). Harvested cells were labeled with RPE-conjugated mouse anti-bovine CD205 (Bio-Rad Laboratories Ltd., Mississauga, ON, Canada; clone: IL-A114) to check the presence of monocytederived dendritic cells and Alexa Fluor 647-conjugated mouse anti-human CD14 (Bio-Rad Laboratories Ltd.; clone: TÜK4) to check the presence of myeloid cells, separately. The labeled samples were analyzed using the BD Accuri C6 flow cytometer (Becton Dickinson, Franklin Lakes, NJ), and the data from the flow cytometer were analyzed by FlowJo V.10 (FlowJo LLC, Ashland, OR). The positive gates were defined based on the fluorescence minus one procedure and the isotype controls were not included based on the absence of any report on the nonspecific binding of fluorochrome used 

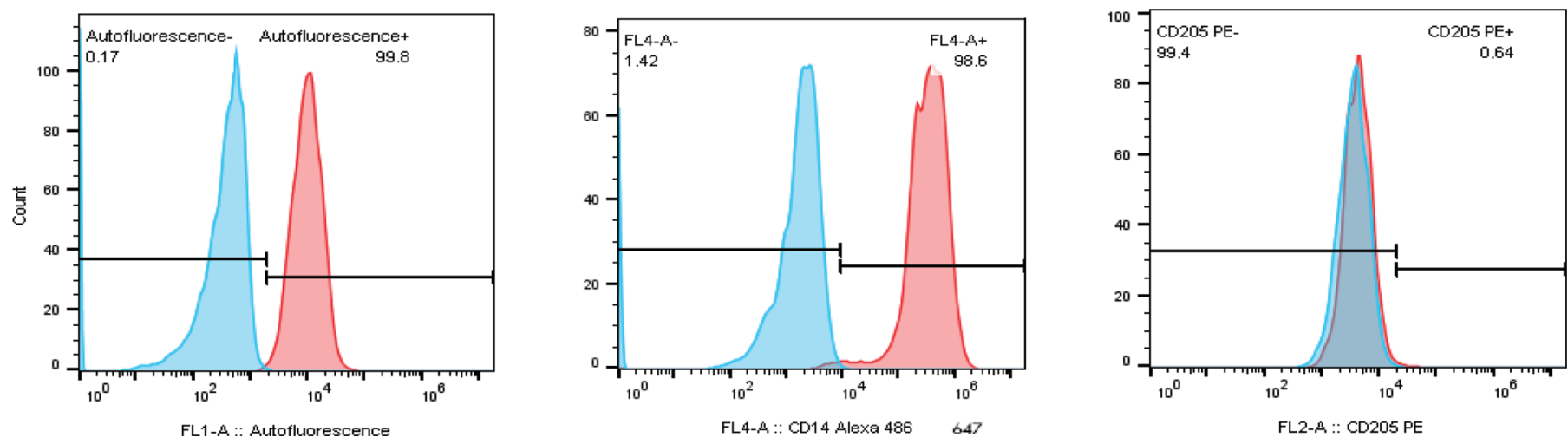

Figure 1. Phenotypic characteristics of harvested cells after $6 \mathrm{~d}$ of in vitro incubation in serum-free medium supplemented with recombinant bovine granulocyte-macrophage colony-stimulating factor. The harvested cells were stained with Alexa Fluor 647 conjugated anti-human CD-14 (Bio-Rad Laboratories Ltd., Mississauga, ON, Canada; clone TÜK4) and phytoerythrin (PE) conjugated anti-bovine CD-205 (Bio-Rad Laboratories Ltd.) separately. The cells were analyzed in BD Accuri C6 cytometer (Becton Dickinson, Franklin Lakes, NJ) against one unstained harvested cell and one unstained blood mononuclear cell as the reference for autofluorescence in a 533/30 filter excited by the blue laser. The data from the flow cytometer were analyzed and graphed using FlowJo (v. 10, FlowJo LLC, Ashland, OR).

in this study (Hulspas et al., 2009). The fluorescence emission of an unlabeled harvested cells was compared with the emission of unlabeled fresh BMC in a 533/30 filter excited by the blue laser to test the auto-fluorescence as an indicator for macrophages (Figure 1).

\section{Phagocytosis}

The MDM from each sample were seeded in 12 wells of an opaque 96-well plate at the concentration of 5 $\times 10^{4}$ cells per well in AIM V medium containing 5 $\mathrm{ng} / \mathrm{mL}$ of GM-CSF and incubated overnight. Each sample was assigned to 2 challenge groups and 2 control groups, each group including 3 wells. Challenge groups were exposed to either pHrodo Green conjugated $E$. coli [multiplicity of infection (MOI) 5] (Thermo Fisher Scientific Inc.) or pHrodo Green conjugated S. aureus (MOI 10; Thermo Fisher Scientific Inc.) for $4 \mathrm{~h}$. One of the control groups was labeled with NucBlue Live ReadyProbes Reagent (Thermo Fisher Scientific Inc.), $20 \mathrm{~min}$ before the reading time. The plates were washed 3 times and the fluorescence intensity (FI) was measured by a microplate reader (Biotek Instruments Inc., Winooski, VT) as an indication of bacterial uptake. pHrodo is fluorescent in an acidic environment and therefore FI represents the bacterial particles in phagolysosomes. The average of FI from each sample (3 wells) in the challenge group was corrected by the average of the same sample in the cell-only control group. Then, the corrected FI for each sample was normalized against the average FI of the control group (12 wells) containing the NucBlue to adjust for the cell number in each sample.

\section{Nitric Oxide Production}

The MDM from each sample were seeded in 3 wells of a 48 -well plate at a density of $2 \times 10^{5}$ cells per well in AIM V Medium containing $5 \mathrm{ng} / \mathrm{mL}$ of GM-CSF and incubated overnight. One well was assigned as the control and the other 2 wells were assigned as treatment groups and were exposed to inactivated E. coli (MOI 5 , determined by titration (MOI 1, 5, 10, and 50) to induce maximum $\mathrm{NO}^{-}$using minimum MOI, the strain was isolated from a mastitic cow by a microbiologist, $\mathrm{P}$. Boerlin, Ontario Veterinary College) or $S$. aureus (MOI 10 , determined by titration (MOI $1,5,10$, and 50) to induce maximum $\mathrm{NO}^{-}$using minimum MOI; Thermo Fisher Scientific Inc.) for $48 \mathrm{~h}$. Supernatant from each well was collected and the concentration of nitric oxide $\left(\mathrm{NO}^{-}\right)$was measured with the Measure-iT HighSensitivity Nitrite Assay Kit (Thermo Fisher Scientific Inc.). The concentration of $\mathrm{NO}^{-}$for every sample in the challenge groups was subtracted by its replicate in the control group.

\section{Statistical Analysis}

On the first data set including samples from 16 cows, 2 functional responses $\left(\mathrm{NO}^{-}\right.$and phagocytosis), and 2 treatments (E. coli and S. aureus), the coefficient of variation was calculated based on dividing the standard deviation of the response ( $\log _{2}$ transformed corrected fluorescence intensity or the nitrate concentration of culture supernatant $48 \mathrm{~h}$ after challenge) by the average of the response for each treatment, separately. In addition, the correlation between the functional character- 
istics of MDM was investigated using Spearman's rank order correlation. These coefficients and their $P$-values were calculated in SAS (version 9.4, SAS Institute Inc., Cary, NC) by employing the PROC CORR procedure.

On the second data set including samples from 58 cows, one functional response $\left(\mathrm{NO}^{-}\right)$, and one treatment (E. coli), the PROC UNIVARIATE in SAS (version 9.4) was used to test the distribution of dependent variable $\left(\mathrm{NO}^{-}\right.$response to E. coli) for normal, log-normal, Weibull, and gamma distributions followed by visual inspection of the quantile-quantile plot. The $P$-values for goodness-of-fit tests based on the empirical distribution function, Anderson-Darling, Cramer-von Mises, and Kolmogorov-Smirnov, for gamma distribution were $>0.50,>0.25$, and $>0.50$, respectively. Therefore, the dependent variable was transformed based on the method described by Krishnamoorthy et al. (2008) and tested again for normality (Figure 2; Krishnamoorthy et al., 2008). The transformed normal data were used in the subsequent statistical analysis. It should be noted that similar procedure was employed on the first data set $(n=16)$. However, no differences were detected on the type of distribution of all 4 functional responses, probably due to small sample size.

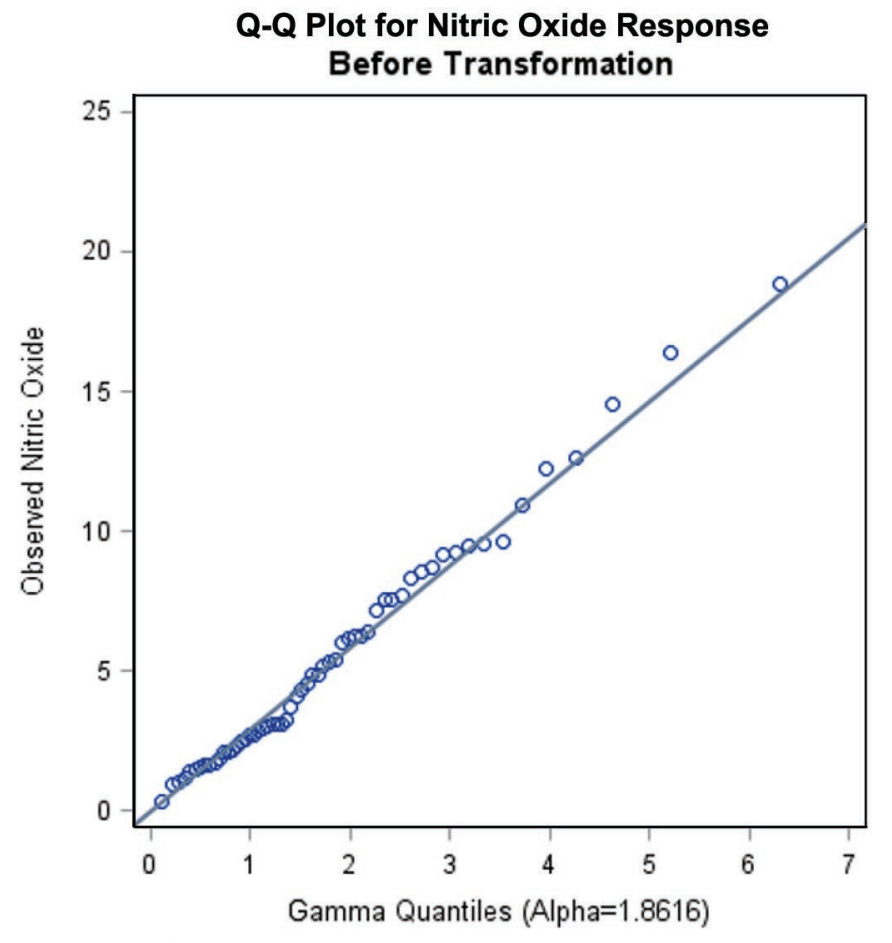

Gamma Line Threshold $=0$, Scale $=2.9279$
Since all the samples were collected from cows in one farm, only the effect of age in month, DIM (classes: $1=0-20 \mathrm{~d}, 2=21-105 \mathrm{~d}, 3=106-235 \mathrm{~d}, 4=>235$ d), days in pregnancy (classes: $1=$ nonpregnant, $2=$ 1-120 d, $3=121-180$ d, $4=181-220$ d; Loker et al., 2009), and sampling group (classes: 1-4 for the first data set and 1-7 for the second data set) were tested by Generalized Linear Model (GLM) procedure in SAS (V 9.4). The statistical model was as follows:

$$
y_{i j k l n}=\mu+\beta \times a_{i}+g_{j}+m_{k}+p_{l}+e_{i j k l n}
$$

where $y_{i j k l n}$ is the phenotypic observation of the $n$th cow [the cubic roots of nitrite concentration of culture supernatant $48 \mathrm{~h}$ after treatment with $E$. coli in the second data set $(\mathrm{n}=58)$ or raw functional measures on the first data set including phagocytosis and nitric oxide response to $E$. coli and $S$. aureus]; $\mu$ is the overall average of the response; $\beta$ is the linear coefficient of the fixed regression on age of the cow ( $a_{i}$; in months); $g_{j}$ is the fixed effect of $j$ th class of sampling group; $m_{k}$ is the fixed effect of $k$ th class of days in milking; $p_{l}$ is the fixed effect of lth class of days in pregnancy; and $e_{i j k l n}$ is the random residual effect.

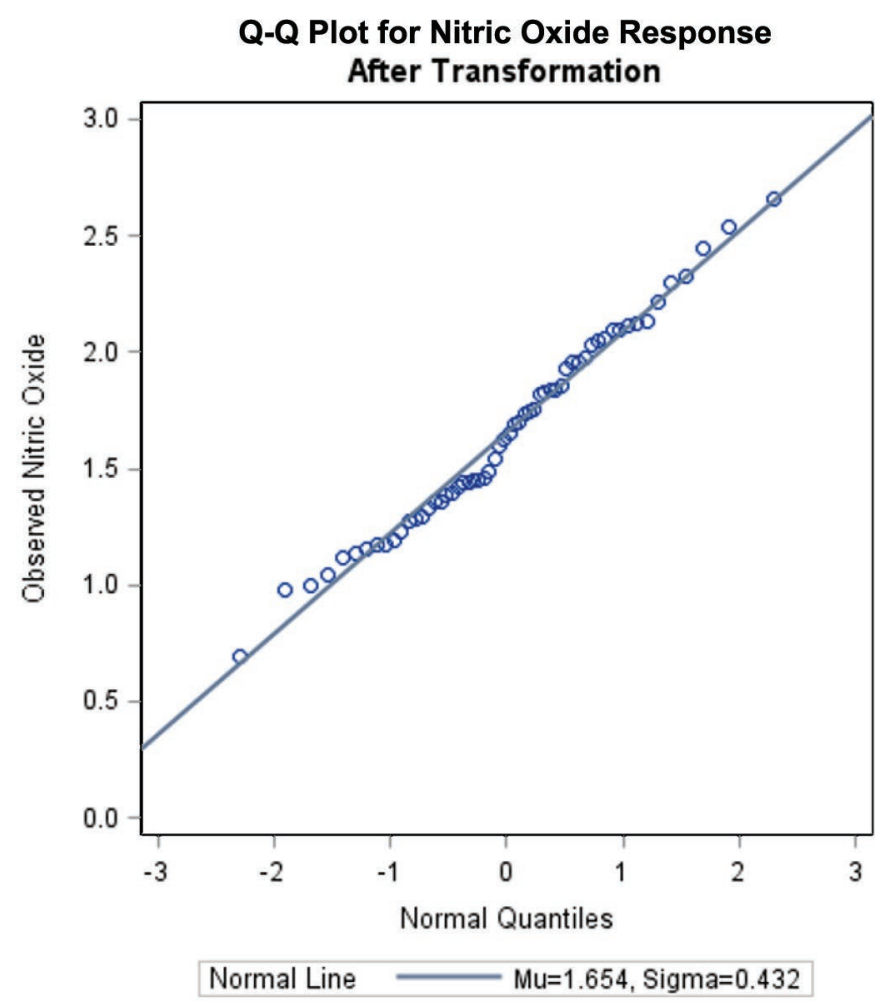

Figure 2. Distribution of nitric oxide response. Nitrite concentration measured by the Measure-iT High-Sensitivity Nitrite Assay Kit (Thermo Fisher Scientific Inc., Mississauga, ON, Canada) in the supernatant of monocyte-derived macrophages $48 \mathrm{~h}$ after treatment with Escherichia coli was transformed based on the method described by Krishnamoorthy et al. (2008). The transformed data were used in subsequent statistical analysis. Q-Q = quantile-quantile. 
Variance components for $\mathrm{NO}^{-}$production were calculated by the REML method in the ASreml package (Ver. 4.1, VSN International) by including the random additive genetic effect of the animal, as in Thompson Crispi et al. (2012b). All available pedigree information was used to calculate the additive genetic relationship matrix fit in the model. Heritability was then calculated dividing the estimated additive genetic variance by the total variance (additive and residual variance).

\section{RESULTS}

\section{Functional Characteristics of MDM}

Monocytes are among the first cells that migrate into the infected tissues and give rise to macrophages. The MDM can eliminate the pathogens via phagocytosis and secretion of bactericidal molecules. In this experiment, the phagocytic ability of bovine MDM and the production of $\mathrm{NO}^{-}$were investigated when MDM were exposed to E. coli and S. aureus. The statistical analysis on the second data set $(\mathrm{n}=58)$ did not show any effect of age $(P$-value $=0.37)$, group of sampling $(P$ value $=0.25)$, DIM $(P$-value $=0.47)$, and pregnancy $(P$-value $=0.73)$ on the production of $\mathrm{NO}^{-}$in response to $E$. coli (model $P$-value $\left.=0.52, \mathrm{R}^{2}=0.21\right)$. Similarly, none of these effects were significant on the 4 functional characteristics of MDM on the first data set $(n=16)$.

Substantial variation was observed among individuals in phagocytic ability and $\mathrm{NO}^{-}$production of MDM (Figures 3 and 4). The coefficient of variation ranged from $33 \%$ in phagocytic ability to $70 \%$ in the production of $\mathrm{NO}^{-}$(inter-assay $\mathrm{CV}$ ranged from 6 to $10 \%$ in phagocytosis and $3-9 \%$ in $\mathrm{NO}^{-}$production). The maximum concentration of $\mathrm{NO}^{-}$in the supernatant of MDM was $42 \mu M$ in response to $E$. coli whereas the highest response to $S$. aureus was $20.5 \mu M$. It should be noted that one cow had the greatest $\mathrm{NO}^{-}$response to both pathogens. Similarly, MDM from another cow produced the lowest $\mathrm{NO}^{-}$in response to $E$. coli and $S$. aureus, 2.7 and $1.8 \mu M$, respectively. The maximum fluorescence intensity (FI) after phagocytosis of $S$. aureus belonged to the same individual that produced the highest amount of $\mathrm{NO}^{-}$. The minimum FI after phagocytosis of $S$. aureus did not belong to the same individual that produced the lowest amount of $\mathrm{NO}^{-}$, but it produced one of the lowest amounts of $\mathrm{NO}^{-}$. To test the repeatability of the results and ensuring the technical assay optimization, $\mathrm{NO}^{-}$response to $E$. coli was chosen as it showed the highest variation among all measured responses. Blood was collected from 4 cows initially sampled and the experiment was repeated independently in a double-blind-like study when the barn and laboratory personnel were not aware of the results of the first experiment. The correlation coefficient between the 2 repeats of 4 cows was 0.97 .

\section{Correlation Between Functional Characteristics of MDM}

The pairwise rank correlation between the 4 characteristics of MDM (phagocytosis and $\mathrm{NO}^{-}$production in response to $E$. coli and $S$. aureus) was investigated using Spearman's rank-order test (Table 1). The highest correlation (0.92) was observed between the production of $\mathrm{NO}^{-}$in response to $E$. coli and $S$. aureus. The correlation between phagocytosis of $E$. coli and $S$. aureus was 0.58 . The lowest correlation (0.53) was observed between the phagocytosis of $S$. aureus and the $\mathrm{NO}^{-}$response to E. coli. These results indicated a strong, significant, and positive correlation among these in vitro functional characteristics of bovine MDM. In addition, the heritability of in vitro $\mathrm{NO}^{-}$production in response to $E$. coli was estimated to be 0.776 .

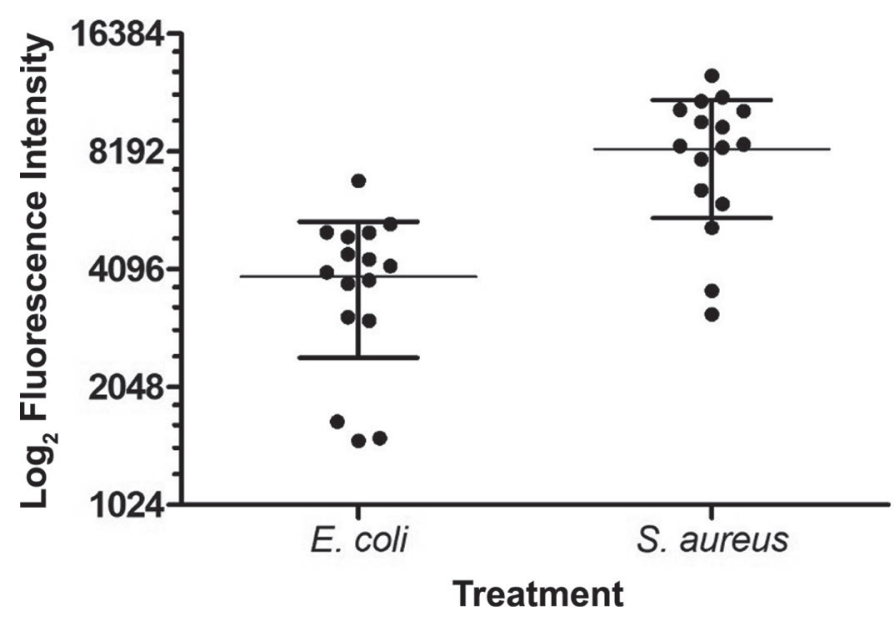

Figure 3. Phagocytic activity of monocyte-derived macrophages in response to in vitro treatment with Escherichia coli and Staphylococcus aureus. Monocyte-derived macrophages were harvested after $6 \mathrm{~d}$ and seeded in 96-well plates at a concentration of $5 \times 10^{4}$ per well. The cells were incubated overnight before treatment. Samples from each individual were treated with pHrodo conjugated $E$. coli [multiplicity of infection (MOI) 5; Thermo Fisher Scientific Inc., Mississauga, ON, Canada] and $S$. aureus (MOI 10), separately. The fluorescence intensity of each sample was corrected based on NucBlue (Thermo Fisher Scientific Inc.) stained control wells with no bacterial treatment. The graph represents $\log _{2}$ transformation of corrected fluorescence intensity as an indication of bacterial uptake. Only relative uptake of each bacterial strain can be compared among the samples in this graph due to the possible differences in saturation of the pHrodo on the surface of each bacterial strain. Each dot represents one individual response, the middle line represents the average in each group, and the error bars represent SD from the mean in each group. 
Table 1. The results of Spearman's rank correlation test $(\mathrm{n}=16)$ between phagocytosis and nitric oxide production of Escherichia coli and Staphylococcus aureus ${ }^{1}$

\begin{tabular}{|c|c|c|c|c|}
\hline Item & $\begin{array}{l}\text { Nitric oxide } \\
\text { response to } E \text {. coli }\end{array}$ & $\begin{array}{l}\text { Phagocytosis } \\
\text { of } E \text {. coli }\end{array}$ & $\begin{array}{l}\text { Nitric oxide } \\
\text { response to } S . \text { aureus }\end{array}$ & $\begin{array}{l}\text { Phagocytosis } \\
\text { of } S \text {. aureus }\end{array}$ \\
\hline $\begin{array}{l}\text { Nitric oxide response to } E \text {. coli } \\
\text { Phagocytosis of } E \text {. coli } \\
\text { Nitric oxide response to } S \text {. aureus } \\
\text { Phagocytosis of } S \text {. aureus }\end{array}$ & 1 & $\begin{array}{l}0.62(0.01) \\
1\end{array}$ & $\begin{array}{l}0.92(<0.01) \\
0.59(0.02) \\
1\end{array}$ & $\begin{array}{l}0.53(0.03) \\
0.58(0.02) \\
0.70(<0.01) \\
1\end{array}$ \\
\hline
\end{tabular}

${ }^{1}$ The Spearman's rank correlation coefficients (rho) between each pair of functional tests are presented in their crossing cell with the $P$-value in parentheses.

\section{DISCUSSION}

Macrophages uniquely play dual roles in the immune system. They are professional antigen presenters, as well as effector cells of the immune system (Mills et al., 2015). Macrophages recognize the presence of pathogens via pathogen recognition receptors. Following recognition, they destroy the pathogens via phagocytosis and producing respiratory burst-derived microbicidal components, such as reactive oxygen and nitrogen species (Murray and Nathan, 1999; Bogdan et al., 2000; Jordao et al., 2007; Flannagan et al., 2015). Recent advances have shown that monocytes are not necessarily giving

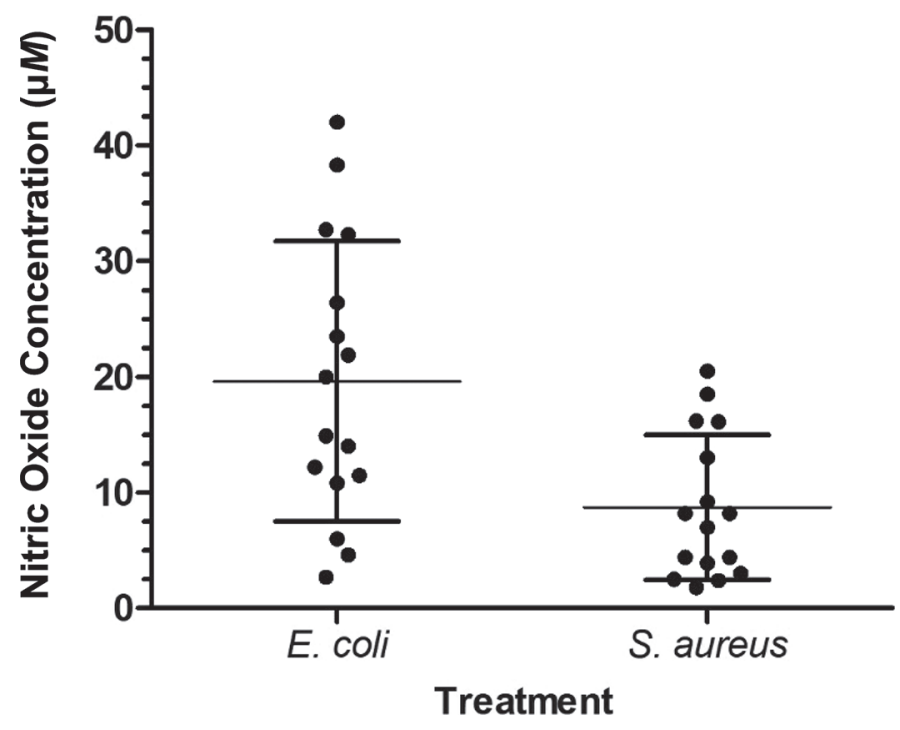

Figure 4. Nitric oxide production of monocyte-derived macrophages in response to in vitro treatment with Escherichia coli and Staphylococcus aureus. Monocyte-derived macrophages were harvested after $6 \mathrm{~d}$ in culture and seeded in 48-well plates at a concentration of $2 \times 10^{5}$ per well. The cells were incubated overnight before bacterial treatment. Samples from each individual were treated with E. coli [multiplicity of infection (MOI) 5] and S. aureus (MOI 10), separately. The concentration of nitric oxide was determined using the MeasureiT High-Sensitivity Nitrite Assay Kit (Thermo Fisher Scientific Inc. Mississauga, ON, Canada) at $48 \mathrm{~h}$ after treatment. Each dot represents one individual response, the middle line represents the average in each group, and the error bars represent SD from the mean in each group. rise to tissue-resident macrophages under steady-state in many tissues (Zigmond and Jung, 2013; Gomez Perdiguero et al., 2015; Hoeffel and Ginhoux, 2015; Franken et al., 2016; Ginhoux et al., 2016). However, during inflammation, proinflammatory macrophages that are transformed from monocytes under the influence of GM-CSF play a significant role in controlling infection (Bain and Mowat, 2014; Ginhoux and Guilliams, 2016; Ginhoux et al., 2016). In in vitro models, blood monocytes can transform to macrophages in the presence of macrophage colony-stimulating factor (M-CSF) or GM-CSF (Italiani and Boraschi, 2017). Adding M-CSF promotes macrophages toward an anti-inflammatory state in which they are also known as M2-like macrophages. These characteristics resemble macrophages under steady-state, but the pitfall in this model is the origin of macrophages, which should not be from blood monocytes. The GM-CSF in cattle, mice, and humans promotes the transformation of blood monocytes toward proinflammatory macrophages, which is also known as M1-like macrophages. These macrophages resemble the macrophages under inflammation which are of the same origin as monocytes (Soehnlein and Lindbom, 2010). Furthermore, under in vitro challenge with pathogens in the presence of M-CSF, MDM receive 2 signals, pro-inflammatory by pathogen-associated molecular pattern (PAMP) and anti-inflammatory by M-CSF. This situation is in contrast to what happens in the host during inflammation. During inflammation, specifically at the peak of inflammation, the local tissue milieu contains GM-CSF along with PAMP from pathogens that stimulate the macrophages in the same direction toward a pro-inflammatory status (Soehnlein and Lindbom, 2010; Becher et al., 2016). Therefore, in the current study, MDM in the presence of GMCSF were chosen as the model. This model seemed to mimic the macrophages in inflamed tissue and exposed to colony stimulating factor that is expressed during inflammation.

Measuring $\mathrm{NO}^{-}$and phagocytosis in in vitro models has been widely used as an indicator of the bactericidal performance of macrophages, but the results are inconsistent between species. A positive correlation between 
the concentration of $\mathrm{NO}^{-}$and bactericidal activity has been reported in rats and chickens but not in mice and cattle (Weiss et al., 2002; Sun et al., 2008; Guimarães et al., 2011; Zhao et al., 2013; Lamont et al., 2014; Akhtar et al., 2016; Qin et al., 2016). Lack of the ability to reliably measure macrophage function, including the production of $\mathrm{NO}^{-}$, seems to be the source of inconsistency. Different research groups have frequently observed the complete or partial failure of macrophages in producing $\mathrm{NO}^{-}$in cultures containing fetal bovine serum (FBS). The MDM from humans, cattle, sheep, goats, badgers, and ferrets in a culture containing FBS were found to be unable to produce a detectable level of $\mathrm{NO}^{-}$or approximately 20 - to 50 -fold less than macrophages from chickens (Denis, 1994; Dumarey et al., 1994; Arias et al., 1997; Ohki et al., 1999; Sacco et al., 2006; Zelnickova et al., 2008; Khalifeh et al., 2009; Guimarães et al., 2011; Azevedo et al., 2016; Bilham et al., 2017; Garcia et al., 2017). The FBS contains a considerable amount of immuno-regulatory cytokines and bioactive molecules. In addition, each batch of FBS can contain different components at different concentrations (Zheng et al., 2006; Beninson and Fleshner, 2015). Transforming growth factor- $\beta$ (TGF- $\beta$ ) is one of the cytokines that is present in FBS in notable concentrations (Oida and Weiner, 2010). The effect of TGF- $\beta$ on macrophages in reducing scavenger receptors and suppressing the production of $\mathrm{NO}^{-}$has been shown in previous studies (Becquet et al., 1994; Ohki et al., 1999; Han et al., 2000; Khalifeh et al., 2009; ReyGiraud et al., 2012; Rath et al., 2014). The similarity and difference between the AA sequence of the isoform 2 of TGF- $\beta$ among cattle (accession number P21214.3), chickens (accession number P30371.1), and humans (accession number P61812.1) can explain the low level of $\mathrm{NO}^{-}$production by human and bovine macrophages in the presence of FBS. Isoform 2 of TGF- $\beta$ in cattle is $99 \%$ identical to its orthologue in humans and only $89 \%$ to that of chickens. Subsequently, the effects of TGF- $\beta$ on human and bovine cells are likely greater compared with chicken. Therefore, in the current study, a serumfree model was developed to measure the functional performance of MDM. The high level of $\mathrm{NO}^{-}$produced by MDM in this culture system and high correlation that was observed between the 2 repeats of $\mathrm{NO}^{-}$production in response to $E$. coli showed the robustness of this serum-free culture system.

The considerable phenotypic variation in this study that was observed in the functional characteristics of MDM among individuals is noteworthy. The statistical analysis revealed no effect of age, DIM, days in pregnancy, and group of sampling on the results of both sample sets (set 1 including 16 cows and set 2 including 58 cows). In addition, the correlation coefficient of $\mathrm{NO}^{-}$ production against $E$. coli in 2 independent experiments was high $(r=0.97)$. Therefore, it was reasoned that the overall variation that was observed in this trait between individuals is likely due to the genetics of the host. This hypothesis aligned with the high heritability (h2 $=0.776$ ) that was estimated for the $\mathrm{NO}^{-}$response to $E$. coli. In addition, the high repeatability and heritability showed that the serum-free culture system and stimulation with GM-CSF is an effective method to evaluate bovine MDM function in vitro and unmask the genetic effects.

Another notable finding of this study is the rank correlation between the functional characteristics of MDM. When these functional traits were compared within one pathogen treatment, the rank correlation was significant, moderately high, and positive (Table 1). These correlating indices of phagocytosis and NO production, 0.61 for E. coli and 0.70 for $S$. aureus, suggest that increased bacterial uptake is providing positive signals that in turn upregulate the pathway of $\mathrm{NO}^{-}$production in response to both pathogen treatments. Correlations between phagocytosis and $\mathrm{NO}^{-}$production have been rarely reported and these reports are mainly limited to in vivo experiments or in vitro experiments only on MDM from chickens and mice (de Matos Macchi et al., 2010; Guimarães et al., 2011).

Additionally, the rank correlation was examined in each functional response (phagocytosis or $\mathrm{NO}^{-}$production) between the 2 bacterial treatments. In this case, the correlation indices were positive, but the difference between the pathogens was large ( 0.58 for phagocytosis versus 0.92 for $\mathrm{NO}^{-}$production). It should be noted that the comparison beyond rank correlation cannot be justified and it was avoided in the current study. The lower FI after phagocytizing E. coli in comparison to $S$. aureus (Figure 3) could be due to the intensity of the fluorochrome after labeling the bacterial particle. However, the difference in ranking correlation can be explained by the mechanisms that macrophages employ to recognize the presence of bacteria and initiate phagocytosis versus production of $\mathrm{NO}^{-}$. Gram-positive and negative bacteria display various sets of PAMP that consist of in-common and gram-specific PAMP. The gram-specific PAMP are recognized by different sets of pathogen recognition receptors (Mogensen, 2009; Martínez-Florensa et al., 2018). However, $\mathrm{NO}^{-}$is produced through a common pathway following activation of macrophages (Moretti and Blander, 2014; Rath et al., 2014). Therefore, the lower correlations for phagocytosis can best be explained by the utilization of different receptors. In addition, it seems genetic control of the receptors that recognize common PAMP, such as scavenger receptors $\mathrm{AI}$ and AII, contribute to the moderate phagocytosis correlation that was observed 
between the 2 bacterial species (Peiser and Gordon, 2001). The significant and positive correlations between these traits also raise the possibility of using only one of the responses as the indicator of functional characteristics of bovine MDM as a method to classify cattle based on MDM function. The strong positive correlation in $\mathrm{NO}^{-}$production (rho $=0.92$ ) along with the notable variation that was observed in $\mathrm{NO}^{-}$response to $E$. coli $(\mathrm{CV}=70 \%)$ suggested it may be suitable to use $\mathrm{NO}^{-}$response to E. coli as a more general indicator of in vitro bovine MDM function. Therefore, only $\mathrm{NO}^{-}$ response against $E$. coli was evaluated in the second sample set $(\mathrm{n}=58$ cows $)$. Although the sample size is small in comparison to in vivo studies on complex traits, when the phenotype is simple, such as gene expression at tissue level in Holstein or $\mathrm{NO}^{-}$response of peritoneal macrophages, approximately 60 samples or less were sufficient to identify expression QTL or heritability (Zídek et al., 2000; Higgins et al., 2018). This observation is probably due to the small effective population size of Holstein $\left(N_{e} \sim 115\right)$ and the simple genetic nature of the measured traits (Stachowicz et al., 2011; Kemper et al., 2016). In a cellular genome-wide association study on human samples, 352 samples were successfully used to investigate the genetic regulation of B lymphocyte response to Salmonella typhi (Alvarez et al., 2017). The $N_{e}$ of Utah residents with Northern and Western European ancestry that samples were collected from in the aforementioned cellular genomewide association study has been estimated to be 10,437 (Park, 2011). Therefore, more samples were required to compensate for the genetic variation in human studies.

The analysis of the phenotypic variation revealed that genetics described $77 \%$ phenotypic variation of this trait (heritability $\mathrm{NO}^{-}$response to E. coli: 0.776 ). The heritability of $\mathrm{NO}^{-}$production of peritoneal macrophages in the in vitro system from mice has also been reported to be very high (broad-sense heritability of 0.81; Zídek et al., 2000). The high heritability does not imply that the environment does not influence MDM function in other contexts, but rather that the culture method employed here is reliable and consistent enough to reveal the additive genetic variation of this trait. Therefore, this method can be further employed along with -omics technology to better understand the genetic control of molecular pathways that shape the response of MDM.

In conclusion, the serum-free culture method for bovine MDM developed in the current study was effective in the evaluation of both phagocytosis and $\mathrm{NO}^{-}$ production. The results of this study are similar to in vivo studies in other species and may provide a feasible approach to measure the activity of inflammatory macrophages in cattle and other large animals in a much less invasive way than broncho-alveolar lavage or tissue biopsies. The strong positive correlations that were observed between phagocytosis and $\mathrm{NO}^{-}$, along with the high heritability of $\mathrm{NO}^{-}$response to $E$. coli, are likely a reflection of the strong contribution of host genetics on the function of MDM, which has been previously reported in mice and humans (Zídek et al., 2000; Motallebipour et al., 2005; Van den Kerkhof et al., 2018). In addition, this model can be used in the future to rank cows based on their MDM function and then to look for disease associations, as well as to better understand the mechanism(s) that determine the magnitude of these responses in MDM following bacterial challenge.

\section{ACKNOWLEDGMENTS}

The authors thank Patrick Boerlin (Department of Pathobiology, Ontario Veterinary College, Guelph, ON, Canada) for providing the E. coli strain and Joseph Okudolo (University of Guelph, Guelph, ON, Canada) for the technical assistant in this study. This research was funded by grants to B. A. Mallard from the Natural Sciences and Engineering Research Council of Canada. This paper is also a contribution to the Food from Thought research program at the University of Guelph, supported by the Canada First Research Excellence Fund from the government of Canada. The funders did not have any role in the design, collection, analysis, and interpretation of data and in writing the manuscript. ME was supported by Natural Sciences and Engineering Research Council of Canada via scholarship.

\section{REFERENCES}

Abel, L., J. Fellay, D. W. Haas, E. Schurr, G. Srikrishna, M. Urbanowski, N. Chaturvedi, S. Srinivasan, D. H. Johnson, and W. R. Bishai. 2018. Genetics of human susceptibility to active and latent tuberculosis: Present knowledge and future perspectives. Lancet Infect. Dis. 18:e64-e75.

Akhtar, R., I. Khan, F. Melzer, H. Neubauer, A. A. Anjum, M. Younus, A. Aslam, and S. Imran. 2016. Differential antibrucella activity of bovine and murine macrophages. Indian J. Exp. Biol. $54: 452-456$.

Alvarez, M. I., L. C. Glover, P. Luo, L. Wang, E. Theusch, S. H. Oehlers, E. M. Walton, T. T. B. Tram, Y.-L. Kuang, J. I. Rotter, C. M. McClean, N. T. Chinh, M. W. Medina, D. M. Tobin, S. J. Dunstan, and D. C. Ko. 2017. Human genetic variation in VAC14 regulates Salmonella invasion and typhoid fever through modulation of cholesterol. Proc. Natl. Acad. Sci. USA 114:E7746-E7755.

Arias, M., J. Zabaleta, J. I. Rodríguez, M. Rojas, S. C. París, and L. F. García. 1997. Failure to induce nitric oxide production by human monocyte-derived macrophages. Manipulation of biochemical pathways. Allergol. Immunopathol. (Madr.) 25:280-288.

Azevedo, M. L. V., N. B. Bonan, G. Dias, F. Brehm, T. M. Steiner, W. M. Souza, A. E. M. Stinghen, F. C. Barreto, S. Elifio-Esposito, R. Pecoits-Filho, and A. N. Moreno-Amaral. 2016. p-Cresyl sulfate affects the oxidative burst, phagocytosis process, and antigen presentation of monocyte-derived macrophages. Toxicol. Lett. 263:1-5. 
Bain, C. C., and A. M. Mowat. 2014. The monocyte-macrophage axis in the intestine. Cell. Immunol. 291:41-48.

Becher, B., S. Tugues, and M. Greter. 2016. GM-CSF: From growth factor to central mediator of tissue inflammation. Immunity 45:963-973.

Becquet, F., Y. Courtois, and O. Goureau. 1994. Nitric oxide decreases in vitro phagocytosis of photoreceptor outer segments by bovine retinal pigmented epithelial cells. J. Cell. Physiol. 159:256-262.

Beninson, L. A., and M. Fleshner. 2015. Exosomes in fetal bovine serum dampen primary macrophage IL-1 $\beta$ response to lipopolysaccharide (LPS) challenge. Immunol. Lett. 163:187-192.

Bilham, K., A. C. Boyd, S. G. Preston, C. D. Buesching, C. Newman, D. W. Macdonald, and A. L. Smith. 2017. Badger macrophages fail to produce nitric oxide, a key anti-mycobacterial effector molecule. Sci. Rep. 7:45470.

Bishop, S. C. 2012. A consideration of resistance and tolerance for ruminant nematode infections. Front. Genet. 3:168.

Bogdan, C., M. Röllinghoff, and A. Diefenbach. 2000. Reactive oxygen and reactive nitrogen intermediates in innate and specific immunity. Curr. Opin. Immunol. 12:64-76.

Clapperton, M., A. B. Diack, O. Matika, E. J. Glass, C. D. Gladney, M. A. Mellencamp, A. Hoste, and S. C. Bishop. 2009. Traits associated with innate and adaptive immunity in pigs: Heritability and associations with performance under different health status conditions. Genet. Sel. Evol. 41:54.

de Matos Macchi, B., J. A. S. Quaresma, A. M. Herculano, M. E. Crespo-López, R. A. DaMatta, and J. L. M. do Nascimento. 2010. Pathogenic action of Plasmodium gallinaceum in chickens: Brain histology and nitric oxide production by blood monocyte-derived macrophages. Vet. Parasitol. 172:16-22.

Denis, M. 1994. Human monocytes/macrophages: NO or no NO? J. Leukoc. Biol. 55:682-684.

Dumarey, C. H., V. Labrousse, N. Rastogi, B. B. Vargaftig, and M. Bachelet. 1994. Selective Mycobacterium avium-induced production of nitric oxide by human monocyte-derived macrophages. J. Leukoc. Biol. 56:36-40.

Dunster, J. L. 2016. The macrophage and its role in inflammation and tissue repair: Mathematical and systems biology approaches. Wiley Interdiscip. Rev. Syst. Biol. Med. 8:87-99.

Flannagan, R. S., B. Heit, and D. E. Heinrichs. 2015. Antimicrobial mechanisms of macrophages and the immune evasion strategies of Staphylococcus aureus. Pathogens 4:826-868.

Franken, L., M. Schiwon, and C. Kurts. 2016. Macrophages: Sentinels and regulators of the immune system. Cell. Microbiol. 18:475-487.

Fuentes-Duculan, J., M. Suárez-Fariñas, L. C. Zaba, K. E. Nograles, K. C. Pierson, H. Mitsui, C. A. Pensabene, J. Kzhyshkowska, J. G. Krueger, and M. A. Lowes. 2010. A subpopulation of CD163positive macrophages is classically activated in psoriasis. J. Invest. Dermatol. 130:2412-2422.

Garcia, M., Y. Qu, C. M. Scholte, D. O'Connor, W. Rounds, and K. M. Moyes. 2017. Regulatory effect of dietary intake of chromium propionate on the response of monocyte-derived macrophages from Holstein cows in mid lactation. J. Dairy Sci.100:6389-6399.

Ginhoux, F., and M. Guilliams. 2016. Tissue-resident macrophage ontogeny and homeostasis. Immunity 44:439-449.

Ginhoux, F., J. L. Schultze, P. J. Murray, J. Ochando, and S. K. Biswas. 2016. New insights into the multidimensional concept of macrophage ontogeny, activation and function. Nat. Immunol. $17: 34-40$.

Gomez Perdiguero, E., K. Klapproth, C. Schulz, K. Busch, E. Azzoni, L. Crozet, H. Garner, C. Trouillet, M. F. de Bruijn, F. Geissmann, and H.-R. Rodewald. 2015. Tissue-resident macrophages originate from yolk-sac-derived erythro-myeloid progenitors. Nature 518:547-551.

Greives, T. J., N. A. Dochtermann, and E. C. Stewart. 2017. Estimating heritable genetic contributions to innate immune and endocrine phenotypic correlations: A need to explore repeatability. Horm. Behav. 88:106-111.

Guimarães, M. C. C., L. V. C. Guillermo, M. F. de R. Matta, S. G. Soares, and R. A. DaMatta. 2011. Macrophages from chickens selected for high antibody response produced more nitric oxide and have greater phagocytic capacity. Vet. Immunol. Immunopathol 140:317-322.

Hamidzadeh, K., S. M. Christensen, E. Dalby, P. Chandrasekaran, and D. M. Mosser. 2017. Macrophages and the recovery from acute and chronic inflammation. Annu. Rev. Physiol. 79:567-592.

Han, J., D. P. Hajjar, J. M. Tauras, J. Feng, A. M. Gotto, and A. C. Nicholson. 2000. Transforming growth factor-beta1 (TGF-beta1) and TGF-beta2 decrease expression of CD36, the type B scavenger receptor, through mitogen-activated protein kinase phosphorylation of peroxisome proliferator-activated receptor-gamma. J. Biol. Chem. 275:1241-1246.

Higgins, M. G., C. Fitzsimons, M. C. McClure, C. McKenna, S. Conroy, D. A. Kenny, M. McGee, S. M. Waters, and D. W. Morris. 2018. GWAS and eQTL analysis identifies a SNP associated with both residual feed intake and GFRA2 expression in beef cattle. Sci. Rep. 8:14301.

Hoeffel, G., and F. Ginhoux. 2015. Ontogeny of tissue-resident macrophages. Front. Immunol. 6:486.

Hulspas, R., M. R. G. O'Gorman, B. L. Wood, J. W. Gratama, and D. R. Sutherland. 2009. Considerations for the control of background fluorescence in clinical flow cytometry. Cytom. Part B Clin. Cytom. 76B:355-364.

Italiani, P., and D. Boraschi. 2017. Development and functional differentiation of tissue-resident versus monocyte-derived macrophages in inflammatory reactions. Results Probl. Cell Differ. 62:23-43.

Jordao, L., C. K. E. Bleck, L. Mayorga, G. Griffiths, and E. Anes. 2007. On the killing of mycobacteria by macrophages. Cell. Microbiol. 10:529-548.

Kemper, K. E., M. D. Littlejohn, T. Lopdell, B. J. Hayes, L. E. Bennett, R. P. Williams, X. Q. Xu, P. M. Visscher, M. J. Carrick, and M. E. Goddard. 2016. Leveraging genetically simple traits to identify small-effect variants for complex phenotypes. BMC Genomics 17:858.

Khalifeh, M. S., A. M. Al-Majali, and J. R. Stabel. 2009. Role of nitric oxide production in dairy cows naturally infected with Mycobacterium avium ssp. paratuberculosis. Vet. Immunol. Immunopathol. 131:97-104.

Ko, D. C., T. J. Urban, U. Pozzoli, A. Ferrer-Admetlla, and L. Pattini. 2013. Understanding human variation in infectious disease susceptibility through clinical and cellular GWAS. PLoS Pathog. 9:e1003424.

Krishnamoorthy, K., T. Mathew, and S. Mukherjee. 2008. Normalbased methods for a gamma distribution. Technometrics 50:69-78.

Lamont, E. A., A. M. Talaat, P. M. Coussens, J. P. Bannantine, Y. T. Grohn, R. Katani, L. Li, V. Kapur, and S. Sreevatsan. 2014. Screening of Mycobacterium avium ssp. paratuberculosis mutants for attenuation in a bovine monocyte-derived macrophage model. Front. Cell. Infect. Microbiol. 4:87.

Loker, S., F. Miglior, J. Bohmanova, J. Jamrozik, and L. R. Schaeffer. 2009. Phenotypic analysis of pregnancy effect on milk, fat, and protein yields of Canadian Ayrshire, Jersey, Brown Swiss, and Guernsey breeds. J. Dairy Sci. 92:1300-1312.

Longley, R., C. Smith, A. Fortin, J. Berghout, B. McMorran, G. Burgio, S. Foote, and P. Gros. 2011. Host resistance to malaria: Using mouse models to explore the host response. Mamm. Genome $22: 32-42$.

Mallard, B. A., M. Emam, M. Paibomesai, K. Thompson-Crispi, and L. Wagter-Lesperance. 2015. Genetic selection of cattle for improved immunity and health. Jpn. J. Vet. Res. 63(Suppl 1):S37S44.

Martínez-Florensa, M., C. Català, M. Velasco-de Andrés, O. Cañadas, V. Fraile-Ágreda, S. Casadó-Llombart, N. Armiger-Borràs, M. Consuegra-Fernández, C. Casals, and F. Lozano. 2018. Conserved bacterial-binding peptides of the scavenger-like human lymphocyte receptor CD6 protect from mouse experimental sepsis. Front. Immunol. 9:627.

McManus, C., T. do P. Paim, C. B. de Melo, B. S. A. F. Brasil, and S. R. Paiva. 2014. Selection methods for resistance to and tolerance of helminths in livestock. Parasite 21:56.

Mills, C. D., L. L. Lenz, and K. Ley. 2015. Macrophages at the fork in the road to health or disease. Front. Immunol. 6:59. 
Min-Oo, G., M. M. Stevenson, A. Fortin, and P. Gros. 2007. Genetic control of host-pathogen interactions in mice. Novartis Found. Symp. 281:156-165.

Minozzi, G., J. L. Williams, A. Stella, F. Strozzi, M. Luini, M. L. Settles, J. F. Taylor, R. H. Whitlock, R. Zanella, and H. L. Neibergs. 2012. Meta-analysis of two genome-wide association studies of bovine paratuberculosis. PLoS One 7:e32578.

Mitchell, A. J., L. C. Pradel, L. Chasson, N. Van Rooijen, G. E. Grau, N. H. Hunt, and G. Chimini. 2010. Technical advance: Autofluorescence as a tool for myeloid cell analysis. J. Leukoc. Biol. $88: 597-603$.

Mogensen, T. H. 2009. Pathogen recognition and inflammatory signaling in innate immune defenses. Clin. Microbiol. Rev. 22:240-273.

Moretti, J., and J. M. Blander. 2014. Insights into phagocytosis-coupled activation of pattern recognition receptors and inflammasomes. Curr. Opin. Immunol. 26:100-110.

Motallebipour, M., A. Rada-Iglesias, M. Jansson, and C. Wadelius. 2005. The promoter of inducible nitric oxide synthase implicated in glaucoma based on genetic analysis and nuclear factor binding. Mol. Vis. 11:950-957.

Murray, H. W., and C. F. Nathan. 1999. Macrophage microbicidal mechanisms in vivo: Reactive nitrogen versus oxygen intermediates in the killing of intracellular visceral Leishmania donovani. J. Exp. Med. 189:741-746.

Njoroge, J. M., L. B. Mitchell, M. Centola, D. Kastner, M. Raffeld, and J. L. Miller. 2001. Characterization of viable autofluorescent macrophages among cultured peripheral blood mononuclear cells. Cytometry 44:38-44.

Ohki, K., F. Amano, S. Yamamoto, and O. Kohashi. 1999. Suppressive effects of serum on the LPS-induced production of nitric oxide and TNF-alpha by a macrophage-like cell line, WEHI-3, are dependent on the structure of polysaccharide chains in LPS. Immunol. Cell Biol. 77:143-152.

Oida, T., and H. L. Weiner. 2010. Depletion of TGF- $\beta$ from fetal bovine serum. J. Immunol. Methods 362:195-198.

Park, L. 2011. Effective population size of current human population. Genet. Res. (Camb.) 93:105-114.

Peiser, L., and S. Gordon. 2001. The function of scavenger receptors expressed by macrophages and their rolein the regulation of inflammation. Microbes Infect. 3:149-159.

Qin, Y., Q. Wang, Y. Zhou, Y. Duan, and Q. Gao. 2016. Inhibition of IFN- $\gamma$-induced nitric oxide dependent antimycobacterial activity by miR-155 and C/EBP $\beta$. Int. J. Mol. Sci. 17:535.

Rath, M., I. Müller, P. Kropf, E. I. Closs, and M. Munder. 2014. Metabolism via arginase or nitric oxide synthase: Two competing arginine pathways in macrophages. Front. Immunol. 5:532.

Rey-Giraud, F., M. Hafner, and C. H. Ries. 2012. In vitro generation of monocyte-derived macrophages under serum-free conditions improves their tumor promoting functions. PLoS One 7:e42656.

Ring, S. C., D. A. Graham, R. G. Sayers, N. Byrne, M. M. Kelleher, M. L. Doherty, and D. P. Berry. 2018. Genetic variability in the humoral immune response to bovine herpesvirus-1 infection in dairy cattle and genetic correlations with performance traits. J. Dairy Sci. 101:6190-6204.

Sacco, R. E., W. R. Waters, K. M. Rudolph, and M. L. Drew. 2006. Comparative nitric oxide production by LPS-stimulated monocyte-derived macrophages from Ovis canadensis and Ovis aries. Comp. Immunol. Microbiol. Infect. Dis. 29:1-11.

Soehnlein, O., and L. Lindbom. 2010. Phagocyte partnership during the onset and resolution of inflammation. Nat. Rev. Immunol. 10:427-439.

Stachowicz, K., M. Sargolzaei, F. Miglior, and F. S. Schenkel. 2011. Rates of inbreeding and genetic diversity in Canadian Holstein and Jersey cattle. J. Dairy Sci. 94:5160-5175.
Sun, S. F., Q. Z. Pan, X. Hui, B. L. Zhang, H. M. Wu, H. Li, W. Xu, Q. Zhang, J. Y. Li, X. M. Deng, J. W. Chen, Z. X. Lian, and N. Li. 2008. Stronger in vitro phagocytosis by monocytes-macrophages is indicative of greater pathogen clearance and antibody levels in vivo. Poult. Sci. 87:1725-1733.

Thompson-Crispi, K. A., B. Hine, M. Quinton, F. Miglior, and B. A. Mallard. 2012a. Short communication: Association of disease incidence and adaptive immune response in Holstein dairy cows. J. Dairy Sci. 95:3888-3893.

Thompson-Crispi, K. A., M. Sargolzaei, R. Ventura, M. Abo-Ismail, F. Miglior, F. Schenkel, and B. A. Mallard. 2014. A genome-wide association study of immune response traits in Canadian Holstein cattle. BMC Genomics 15:559.

Thompson-Crispi, K. A., A. Sewalem, F. Miglior, and B. A. Mallard. 2012b. Genetic parameters of adaptive immune response traits in Canadian Holsteins. J. Dairy Sci. 95:401-409.

Van den Kerkhof, M., L. Van Bockstal, J. F. Gielis, P. Delputte, P. Cos, L. Maes, G. Caljon, and S. Hendrickx. 2018. Impact of primary mouse macrophage cell types on Leishmania infection and in vitro drug susceptibility. Parasitol. Res. 117:3601-3612.

Wang, L., K. J. Pittman, J. R. Barker, R. E. Salinas, I. B. Stanaway G. D. Williams, R. J. Carroll, T. Balmat, A. Ingham, A. M. Gopalakrishnan, K. D. Gibbs, A. L. Antonia, J. Heitman, S. C. Lee, G. P. Jarvik, J. C. Denny, S. M. Horner, M. R. DeLong, R. H Valdivia, D. R. Crosslin, D. C. Ko, and D. C. Ko. 2018. An atlas of genetic variation linking pathogen-induced cellular traits to human disease. Cell Host Microbe 24:308-323.

Weiss, D. J., O. A. Evanson, A. Moritz, M. Q. Deng, and M. S. Abrahamsen. 2002. Differential responses of bovine macrophages to Mycobacterium avium ssp. paratuberculosis and Mycobacterium avium ssp. avium. Infect. Immun. 70:5556-5561.

Zelnickova, P., J. Matiasovic, B. Pavlova, H. Kudlackova, F. Kovaru, and M. Faldyna. 2008. Quantitative nitric oxide production by rat, bovine and porcine macrophages. Nitric Oxide 19:36-41.

Zerbino, D. R., P. Achuthan, W. Akanni, M. R. Amode, D. Barrell J. Bhai, K. Billis, C. Cummins, A. Gall, C. G. Girón, L. Gil, L. Gordon, L. Haggerty, E. Haskell, T. Hourlier, O. G. Izuogu, S. H. Janacek, T. Juettemann, J. K. To, M. R. Laird, I. Lavidas, Z. Liu, J. E. Loveland, T. Maurel, W. McLaren, B. Moore, J. Mudge, D. N. Murphy, V. Newman, M. Nuhn, D. Ogeh, C. K. Ong, A. Parker, M. Patricio, H. S. Riat, H. Schuilenburg, D. Sheppard, H. Sparrow, K. Taylor, A. Thormann, A. Vullo, B. Walts, A. Zadissa, A. Frankish, S. E. Hunt, M. Kostadima, N. Langridge, F. J. Martin, M. Muffato, E. Perry, M. Ruffier, D. M. Staines, S. J. Trevanion, B. L. Aken, F. Cunningham, A. Yates, and P. Flicek. 2018. Ensembl 2018. Nucleic Acids Res. 46:D754-D761.

Zhao, Z.-J., J. Zhang, J. Wei, Z. Li, T. Wang, S.-Q. Yi, J.-L. Shen, T.-B. Yang, G. Hide, and Z.-R. Lun. 2013. Lower expression of inducible nitric oxide synthase and higher expression of arginase in rat alveolar macrophages are linked to their susceptibility to Toxoplasma gondii infection. PLoS One 8:e63650.

Zheng, X., H. Baker, W. S. Hancock, F. Fawaz, M. McCaman, and E. Pungor. 2006. Proteomic analysis for the assessment of different lots of fetal bovine serum as a raw material for cell culture. Part IV. Application of proteomics to the manufacture of biological drugs. Biotechnol. Prog. 22:1294-1300.

Zídek, Z., D. Franková, and M. Boubelík. 2000. Genetic variation in in vitro cytokine-induced production of nitric oxide by murine peritoneal macrophages. Pharmacogenetics 10:493-501.

Zigmond, E., and S. Jung. 2013. Intestinal macrophages: Well educated exceptions from the rule. Trends Immunol. 34:162-168. 